\title{
Study of the type 2 diabetic patients' status of care and control in rural areas of Ardabil Province in 2017, Iran
}

\section{ABSTRACT}

Introduction. Diabetes mellitus includes a group of metabolic disorders diagnosed by increased serum glucose concentration. It causes major changes in most systems of the body, which lead to acute and chronic complications of the disease which results in disability, medical costs, and high mortality. This research was conducted to assess type 2 diabetic patients' status of care and control in rural areas of Ardebil province in order to improve the care indicators by presenting results to regional authorities.

Materials and methods. In this cross-sectional descriptive-analytic study, 360 patients identified as type 2 diabetes in rural areas of Ardabil province were selected through cluster sampling and data was collected through a questionnaire by interviewing patients and reviewing their files, and 3 categories of demographic, clinical and socio-economic factors were studied. The data was analyzed by Chi-square and Friedman statistical tests using SPSS software version 20.

Results. According to Chi-square test, there was a significant relationship between the patients' marital status ( $p=0.032$ ) and their complications of diabetes ( $p=0.10)$ and the level of care and between the number of patients' family members $(p=0.001)$ and body mass index $(p=0.006)$ and the level of control and also, between the use of ordered drug by patient and

Address for correspondence:

Forough Farzollahpour

Department of Midwifery, Khalkhal University of Medical Sciences

Khalkhal, Iran

e-mail: almas.submission@gmail.com

Clinical Diabetology 2019, 8, 3, 161-166

DOI: $10.5603 /$ DK.2019.0011

Received: 27.02.2019

Accepted: 09.03.2019 the level of care and control ( $p=0.003$ ). The results of the mean ranking based on the Friedman statistical test showed that the age variable has the highest mean score and the greatest impact on the care and control of the disease and the lowest score and impact was of the nutrition education variable.

Conclusion. This study revealed that in order to control the complications of the disease, lifestyle changes, dietary observation, weight control, ordered drug use and self-care programs are very effective. (Clin Diabetol 2019; 8, 3: 161-166)

Key words: care and control, type 2 diabetes, rural areas

\section{Introduction}

Diabetes mellitus includes a group of metabolic disorders that all are diagnosed by increased serum glucose concentration. Different types of the disease are due to various reasons caused by genetic factors, environmental factors and lifestyle [1]. 59 percent of all deaths in the world and 46 percent of the burden of illness are due to non-infectious diseases, and statistics demonstrate an increase in the prevalence of these diseases. The damage of these diseases and their costs for health systems has always been a serious and strong incentive to design and implement prevention programs at various levels [2]. Despite the advances of medical science, not only the spread of the disease is not reduced but also it is increasing every day [3]. Diabetes is an ice burg-like disease that afflicts around 30 million people around the world and its abundance in most adult societies is between 2 to 5 percent [4]. According to the World Health Organization classifi- 
cation, variety of diabetes includes type 1 or insulin dependent, type 2 or non-insulin dependent which is the most common type of diabetes, gestational diabetes and specific types of diabetes [5]. More than $90 \%$ of diabetic patients are suffering from type 2 diabetes [6]. Type 2 is a disease with severe difficulties and complications and problems both in terms of the cost of care and financial burden of disability [7]. Due to statistics and global increasing trend, the World Health Organization (WHO) has reported diabetes as a latent epidemic, and since 1993, called all countries in the world to cope with this epidemic. The prevalence of diabetes, especially type 2 , has increased significantly in recent decades [1]. Recent studies in Iran reported prevalence of $5.5 \%$ and $7.7 \%$ of diabetes among adults [8]. Diabetes causes dangerous complications such as atherosclerosis, retinopathy, and nephropathy leading to renal failure, and peripheral neuropathy with the risk of complications of diabetic foot [9]. Progress of complications and the cost of treatment among diabetic patients are mainly due to inappropriate blood glucose control [10]. According to the studies, half of the type 2 diabetic patients are unaware of their disease and are diagnosed totally random. Almost in all health systems, a diabetic patient costs 2 to 4 times more than a non-diabetic person. Its direct costs takes $1.5-2.5 \%$ of the total health budget and its indirect costs (due to the cost of hospitalization, the occurrence and exacerbation of chronic complications) are manifold and its subtle costs are unpredictable. On the other hand, while preventing complications of diabetes, many costs and deaths can be reduced by proper care and implementing preventive measures regarding complications of the disease, such as proper control of blood glucose, proper nutrition and exercise among diabetic patients [5].

As a developing country, Iran faces a massive and salient increase in the population of diabetic patients by approximate increase of $195 \%$ compared with the current prevalence [8]. In Iran, in 1996, after a comprehensive review, the national program to prevent and control the diabetes was designed to integrate into the health network system. The program was aimed to combat this global problem through primary, secondary and tertiary preventive measures [5]. In addition to drug use and diet observance, among the factors influencing blood glucose control, the role of other variables such as age, sex, economic status, educational level and family factors in controlling blood glucose cannot be ignored. Identification of these factors can be effective in designing necessary interventions to control blood glucose [11]. Among the factors influencing on the control of diabetes, there are patient related factors, such as the socio-economic class and the patient's lifestyle, and the duration of engagement and awareness and attitude toward diabetes and physician related factors such as knowledge, personality and counseling style and facilities of the health center are mentioned. The evidence suggests that controlling blood glucose can reduce the risk of debilitating and even lethal complications of diabetes [12]. One of the most important goals in treatment of diabetic patients is to achieve proper blood glucose control. An important indicator of blood glucose control is glycosylated hemoglobin $\left(\mathrm{HbA}_{1 \mathrm{c}}\right)$, and in fact the best target for controlling and treating diabetes is to maintain the level of glycosylated hemoglobin in normal range. Unlike other indicators, this indicator is not affected by daily fluctuations of blood glucose [11].

This research was conducted to investigate the effective factors of management to control of type 2 diabetic patients in rural areas of Ardabil province in order to provide results for the regional authorities to improve care indicators.

\section{Method}

This is a descriptive-analytic study that has been conducted to study type 2 diabetic patients' status of care and control in rural areas of Ardebil province. The samples of this study were diabetic patients from rural areas of Ardabil province which the number of them in these areas was 5,608. The sample size was determined 360 by sample estimation formula. In this research, the patients were divided into 10 clusters and clusters were assigned relative to all patients in each city, then, the clusters considered for each city were randomly allocated among the covered villages by simple random sampling and the required information was collected by interviewing and reviewing their files thorough a questionnaire and the results were analyzed by Chi-square and Friedman statistic tests using version 20 of the SPSS software.

\section{Findings}

The results of the Chi-square test indicated that there is no significant relationship between gender, age, educational level, occupation, income, hypertension, smoking, vegetable consumption, exercise, nutrition education, training the complications of the disease, and the level of care and control of diabetes, but there is a significant relationship between the marital status of the patients and the complications of diabetes and the level of care, but there is no significant relationship with the level of diabetes control. There is also a significant relationship between the patients' number of family members, body mass index and the family history of diabetes and the diabetes control level, but no 
Table 1. Demographic characteristics of the studied samples regarding care and control

\begin{tabular}{|c|c|c|c|c|}
\hline Variable & Care & $p$ & Control & $p$ \\
\hline \multicolumn{5}{|l|}{ Gender } \\
\hline Male & 93 & NS & 28 & NS \\
\hline Female & 202 & & 73 & \\
\hline \multicolumn{5}{|l|}{ Age } \\
\hline $39-30$ & 19 & NS & 4 & NS \\
\hline $49-40$ & 37 & & 12 & \\
\hline $59-50$ & 106 & & 40 & \\
\hline $69-60$ & 71 & & 29 & \\
\hline Above 70 & 62 & & 16 & \\
\hline \multicolumn{5}{|l|}{ Marital status } \\
\hline Single & 21 & $p=0.03$ & 274 & NS \\
\hline Married & 9 & & 92 & \\
\hline \multicolumn{5}{|l|}{ Education } \\
\hline Illiterate & 224 & NS & 77 & NS \\
\hline High school diploma & 63 & & 21 & \\
\hline University graduated & 8 & & 3 & \\
\hline \multicolumn{5}{|l|}{ Occupation } \\
\hline Housewife & 196 & NS & 70 & NS \\
\hline Farmer & 67 & & 23 & \\
\hline Manual worker & 13 & & 4 & \\
\hline Unemployed & 16 & & 3 & \\
\hline Employee & 3 & & 1 & \\
\hline \multicolumn{5}{|l|}{ Family size } \\
\hline Below 5 & 186 & NS & 78 & $p=0.001$ \\
\hline 5 to 10 & 106 & & 23 & \\
\hline 10 to 15 & 3 & & 0 & \\
\hline
\end{tabular}

NS - not significant

significant relationship with the level of care, and finally, there is a significant relationship between the use of ordered drug and the level of diabetes care and control.

The results of Friedman statistical test revealed that there is a relationship between the care and control of diabetes mellitus and the patients' epidemiologic factors, and also the results of mean ranking showed that the highest mean scores of the patients' questionnaire included age, body mass index, marital status, complications of illness, smoking, drug discontinuation, incomes, and consumption of vegetables respectively which had the greatest impact on the control and care of the disease, and the mean of the nutrition education variable has the lowest score (Tables 1-4).

\section{Discussion}

In the study by Shiva Heidari et al., the mean glycated hemoglobin of the participants was $9.4 \pm 0.9$ and most units (57.4\%) had no favorable blood glucose control. The results showed a significant relationship between blood glucose control and the marital status $(p<0.0001)$, economic status $(p=0.003)$, duration of diabetes ( $p=0.03)$, home glucose test $(p=0.01)$, family structure $(p=0.01)$ and family support $(p<$ 0.0001 ). According to the research, the results of Friedman statistical test showed that age, body mass index, marital status, complications of disease, smoking, drug discontinuation, income, and consumption of vegetables cause the greatest impact on the care and control of the disease, which indicates the consistency of some of these results and the results of the study by Heidari et al. [11].

Gholamreza Sharifirad in a descriptive-analytic study showed that most subjects had one outcome, cardiac complications (22.2\%), two outcomes, cardiovascular and ophthalmic complications (12.7\%) and three outcomes, cardiac and ophthalmic complications, and feet wound (14\%). The majority of subjects (50\%) experienced one outcome of diabetes, while $33.6 \%$ had two outcomes and $16.4 \%$ had three or more. In the present study, there was at least one complication in most patients [13]. 
Table 2. Clinical characteristics of the studied samples regarding care and control

\begin{tabular}{|c|c|c|c|c|}
\hline Variable & Care & $\mathrm{p}$ & Control & $p$ \\
\hline \multicolumn{5}{|l|}{ Body mass index } \\
\hline Thin & 12 & NS & 6 & $p=0.006$ \\
\hline Normal weight & 71 & & 21 & \\
\hline Overweight & 131 & & 39 & \\
\hline Obesity & 81 & & 35 & \\
\hline Presence of diabetes in family & 152 & NS & 40 & $p=0$ \\
\hline Hypertension & 132 & NS & 45 & NS \\
\hline \multicolumn{5}{|l|}{ Presence of complications } \\
\hline Ophthalmic & 28 & $p=0.01$ & 8 & NS \\
\hline Renal & 16 & & 8 & \\
\hline Cardiac & 29 & & 12 & \\
\hline Late wound healing & 39 & & 9 & \\
\hline No complication & 183 & & 64 & \\
\hline Ordered drug use & 262 & NS & 93 & $p=0$ \\
\hline Nutrition education & 269 & NS & 95 & NS \\
\hline Training complications of the disease & 276 & NS & 97 & NS \\
\hline Instructing drug use & 274 & NS & 93 & NS \\
\hline
\end{tabular}

NS - not significant

Table 3. Socioeconomic characteristics of the studied samples regarding care and control

\begin{tabular}{|c|c|c|c|c|}
\hline Variable & Care & $p$ & Control & $p$ \\
\hline \multicolumn{5}{|l|}{ Income } \\
\hline Low & 94 & NS & 30 & NS \\
\hline medium & 188 & & 64 & \\
\hline High & 13 & & 7 & \\
\hline Smoking & 42 & NS & 11 & NS \\
\hline \multicolumn{5}{|c|}{ Vegetable consumption } \\
\hline Below $100 \mathrm{~g}$ & 101 & NS & 32 & NS \\
\hline $20-100 \mathrm{~g}$ & 171 & & 57 & \\
\hline $200-400 \mathrm{~g}$ & 23 & & 12 & \\
\hline Exercise & 191 & NS & 66 & NS \\
\hline
\end{tabular}

NS - not significant

The results of the study by Nader Esmaeilnasab et al., Showed that according to the results of the measurement of hemoglobin glycosylated, $26.8 \%$ of the patients had proper control (glycated hemoglobin less than 6) and $73.2 \%$ of moderate control (glycated hemoglobin 6 to 8 ) or weak (glycated hemoglobin above 8). There was no significant relationship between fasting blood glucose and gender, age, body mass index, duration of onset and duration of referral and insulin injection, but there was a significant relationship between fasting blood glucose and patients' education and occupation. However, in the results of the present study, in most patients, glycated hemoglobin levels were above 7 , despite the ordered care by practical nurse and the physician and were classified as uncontrolled patients in accordance with the guidelines of the country. Also, contrary to the results of our study, Nader Esmaeilnasab et al., found a significant relationship between body mass index and control of disease [12].

In 2013, Fosse-Edorh et al., in a cross-sectional study showed that type 2 diabetes in women was correlated with age, body mass index and occupation, but was not related to the level of education. In men, type 2 diabetes was not associated with the birth place. According to the present study, the majority of studied patients were female [14]. 
Table 4. The mean ranking of the impact of patients' variables on the control and management of diabetes based on Friedman test

\begin{tabular}{lcc}
\hline Rank & Variable & Mean score \\
\hline 1 & Age & 38.93 \\
2 & Body mass index & 38.27 \\
3 & Marital status & 30.75 \\
4 & Suffering from complications & 30.15 \\
5 & Smoking & 28.98 \\
6 & Discontinue medication & 28.73 \\
7 & Income & 27.27 \\
8 & Vegetable consumption & 26.37 \\
9 & Hypertension & 24.07 \\
10 & Sex & 21.17 \\
11 & Family history of diabetes & 20.60 \\
12 & Family size & 19.96 \\
13 & Occupation & 19.38 \\
14 & Exercise & 18.16 \\
15 & Education & 15.18 \\
16 & Residence & 13.91 \\
17 & Regular visits for caring & 13.88 \\
18 & Instructing drug use & 13.85 \\
19 & Training of the complications & 13.58 \\
20 & Nutrition education & 12.98 \\
\hline
\end{tabular}

A study by Zeng Bin et al., in 2014, revealed that the most associated factors of self-management of diabetes included five groups: social characteristics, behavioral and psychological characteristics, social support, language barriers, and cultural characteristics. Also, according to the results, factors such as quality of life, glycated hemoglobin, and blood pressure and other cardiovascular risk factors were potentially associated with improving self-management of diabetes. According to the results of the research, there is a significant relationship between the use of the ordered drug by patient and the level of diabetes care and control, which suggests patients' self-management in this regard [15].

The study by Kumar and Sandhya, in 2018, about blood glucose control, lipid profile and blood pressure among type 2 diabetic patients in northern Kerala, India, showed that the high average of blood glucose levels, resulted in a predictable increase of vascular disease, which In turn, affects the quality of health and efficiency. Generally, this study suggests that individual and community economic growth for therapeutic interventions to improve glycemic control can reduce the risk of cardiovascular and fungal diseases. The study showed that there is a need for more drugs, better strategies and more emphasis on glycemic control, to increase the level of control on diabetes which was undesirable in Kerala [16].
The study by Sanjoy K Paul et al., in 2018, found that among type 2 diabetic patients with normal weight, the white European population had a significantly higher incidence of cardiovascular disease than South Asian populations. Overweight and obese diabetic patients from South Asia and the Caribbean region had the same prevalence of cardiovascular disease, while the white European population that was obese had a significant higher prevalence of cardiovascular disease. Among patients of South Asia and South Africa who were obese, the risk of major cardiovascular events was significantly higher among overweight people compared to European white peers during a mean period of 7 years follow up. However, there were similar levels of risk for the white population of Europe and South Asia in normal weight. The risk of developing chronic kidney disease among European and South Asian population with a BMI $\geq 25$ was high and the same, while among the African-Caribbean population only overweight patients were at high risk of chronic kidney disease [17].

\section{Conclusion}

The mentioned studies, as well as other researches, indicate that factors such as age, body mass index, marital status, economic status, and complications of the disease affects the control of the disease. The results of the distribution of variables by Kolmogorov Smirnov test showed that the distribution of variables was not normal ( $p=0.05)$ and also, in order to determine the reliability of the questionnaire, Cronbach's alpha of SPSS software was used, and the alpha value showed that the reliability of the questionnaire was decent ( $\alpha=0.7$ ). Investigating the results of the chi-square test of the patients' questionnaire showed that there is no significant relationship between the gender, age, education, occupation, income, hypertension, smoking, vegetable consumption, exercise, nutrition education, drug use training, and the level of care and control of diabetes, but there is a significant relationship between the patients' marital status and the complications of diabetes, and the level of care, despite any significant relationship with the level of diabetes control. Also, there is a significant relationship between the number of family members and the body mass index and the diabetes control, but, there is no significant relationship with a level of care and finally, there is a significant relationship between the use of the ordered drug by the patient and the level of diabetes care and control. The results of Friedman statistical test showed that there is relationship between the care and control of diabetes and patients' epidemiologic factors, and also the results of mean ranking, revealed that the highest mean scores 
in the patients' questionnaire were age, body mass index, marital status, smoking, The amount of income and consumption of vegetables respectively, which has caused the greatest impact on the control and care of the disease and the mean of the nutrition education variable is the lowest score.

\section{Conflict of interest}

The authors affirm that there is no conflict of interest involved in the writing of this paper.

\section{REFERENCES}

1. Adjah EO, Ray K, Paul S. Ethnicity-specific association of BMI levels at diagnosis of type 2 diabetes with cardiovascular disease and all-cause mortality risk. Acta Diabetologica. 2018; 56(1): 87-96, doi: 10.1007/s00592-018-1219-7.

2. Woodman RJ, Mori TA, Burke V, et al. Effects of purified eicosapentaenoic and docosahexaenoic acids on glycemic control, blood pressure, and serum lipids in type 2 diabetic patients with treated hypertension. Am J Clin Nutr. 2002; 76(5): 1007-1015, doi: 10.1093/ajcn/76.5.1007, indexed in Pubmed: 12399272.

3. Dunstan DW, Daly RM, Owen N, et al. High-intensity resistance training improves glycemic control in older patients with type 2 diabetes. Diabetes Care. 2002; 25(10): 1729-1736, doi: 10.2337/ /diacare.25.10.1729.

4. Lan J, Zhao Y, Dong F, et al. Meta-analysis of the effect and safety of berberine in the treatment of type 2 diabetes mellitus, hyperlipemia and hypertension. J Ethnopharmacol. 2015; 161: 69-81, doi: 10.1016/j.jep.2014.09.049, indexed in Pubmed: 25498346.

5. Marso SP, Daniels GH, Brown-Frandsen K, et al. LEADER Steering Committee, LEADER Trial Investigators. Liraglutide and cardiovascular outcomes in type 2 diabetes. N Engl J Med. 2016; 375(4): 311-322, doi: 10.1056/NEJMoa1603827, indexed in Pubmed: 27295427.

6. Levelt E, Pavlides M, Banerjee R, et al. Ectopic and visceral fat deposition in Lean and obese patients with type 2 diabetes. J Am Coll Cardiol. 2016; 68(1): 53-63, doi: 10.1016/j.jacc.2016.03.597, indexed in Pubmed: 27364051.

7. DeFronzo RA, Ferrannini E, Groop L, et al. Type 2 diabetes mellitus. Nat Rev Dis Primers. 2015; 1: 15019, doi: 10.1038/nrdp.2015.19, indexed in Pubmed: 27189025.
8. Wulffelé MG, Kooy A, de Zeeuw $D$, et al. The effect of metformin on blood pressure, plasma cholesterol and triglycerides in type 2 diabetes mellitus: a systematic review. J Intern Med. 2004; 256(1): 1-14, doi: 10.1111/j.1365-2796.2004.01328.x, indexed in Pubmed: 15189360.

9. Gaede P, Vedel P, Larsen N, et al. Multifactorial intervention and cardiovascular disease in patients with type 2 diabetes. N Engl J Med. 2003; 348(5): 383-393, doi: 10.1056/NEJMoa021778, indexed in Pubmed: 12556541.

10. Hodgson JM, Watts GF, Playford DA, et al. Coenzyme Q10 improves blood pressure and glycaemic control: a controlled trial in subjects with type 2 diabetes. Eur J Clin Nutr. 2002; 56(11): 1137-1142, doi: 10.1038/sj.ejcn.1601464, indexed in Pubmed: 12428181.

11. Colhoun $H$, Betteridge $D$, Durrington $P$, et al. Primary prevention of cardiovascular disease with atorvastatin in type 2 diabetes in the Collaborative Atorvastatin Diabetes Study (CARDS): multicentre randomised placebo-controlled trial. The Lancet. 2004; 364(9435): 685-696, doi: 10.1016/s0140-6736(04)16895-5.

12. Scheen A, Finer $N$, Hollander $P$, et al. Efficacy and tolerability of rimonabant in overweight or obese patients with type 2 diabetes: a randomised controlled study. The Lancet. 2006; 368(9548): 1660-1672, doi: 10.1016/s0140-6736(06)69571-8.

13. Kemp TM, Barr E, Zimmet PZ, et al. Glucose, Lipid, and Blood Pressure Control in Australian Adults With Type 2 Diabetes: The 1999-2000 AusDiab. Diabetes Care. 2005; 28(6): 1490-1492. doi: 10.2337/diacare.28.6.1490

14. Fosse-Edorh S, Fagot-Campagna A, Detournay B, et al. Type 2 diabetes prevalence, health status and quality of care among the North African immigrant population living in France. Diabetes Metab. 2014; 40(2): 143-150, doi: 10.1016/j.diabet.2013.11.005, indexed in Pubmed: 24447725.

15. Zeng B, Sun W, Gary RA, et al. Towards a conceptual model of diabetes self-management among Chinese immigrants in the United States. Int J Environ Res Public Health. 2014; 11(7): 6727-6742, doi: 10.3390/ijerph110706727, indexed in Pubmed: 24978878.

16. Kumar SP, Sandhya AM. A study on the glycemic, lipid and blood pressure control among the type 2 diabetes patients of north Kerala, India. Indian Heart J. 2018; 70(4): 482-485, doi: 10.1016/j. ihj.2017.10.007, indexed in Pubmed: 30170640.

17. Adjah ESO, Bellary S, Hanif W, et al. Prevalence and incidence of complications at diagnosis of T2DM and during follow-up by BMI and ethnicity: a matched case-control analysis. Cardiovasc Diabetol. 2018; 17(1): 70, doi: 10.1186/s12933-018-0712-1, indexed in Pubmed: 29764436. 